Received Date : 12-Aug-2016

Revised Date : 21-Sep-2016

Accepted Date : 11-Oct-2016

Article type : Ordinary Papers

\title{
RB but not R-HCVAD is a feasible induction regimen prior to auto-HCT in frontline MCL: results of SWOG Study S1106
}

Robert W. Chen ${ }^{1}$, Hongli Li ${ }^{2}$, Steven H. Bernstein ${ }^{3}$, Samir Kahwash ${ }^{4}$, Lisa M. Rimsza ${ }^{5}$, Stephen J. Forman ${ }^{6}$, Louis Constine ${ }^{7}$, Thomas C. Shea ${ }^{8}$, Amanda F. Cashen ${ }^{9}$, Kristie A. Blum $^{10}$, Timothy S. Fenske ${ }^{11}$, Paul M. Barr ${ }^{12}$, Tycel Phillips ${ }^{13}$, Michael Leblanc ${ }^{2}$, Richard I. Fisher ${ }^{14}$, Bruce D. Cheson ${ }^{15}$, Sonali M. Smith ${ }^{16}$, Malek Faham ${ }^{17}$, Jennifer Wilkins ${ }^{17}$, John P. Leonard ${ }^{18}$, Brad S. Kahl ${ }^{19}$, Jonathan W. Friedberg ${ }^{20}$

${ }^{1}$ City of Hope, Duarte, CA; ${ }^{2}$ SWOG Statistical Center, Seattle, WA; 3ames P. Wilmot Cancer Center, University of Rochester School of Medicine and Dentistry, Rochester, $\mathrm{NY} ;{ }^{4}$ Nationwide Children's Hospital, Columbus, $\mathrm{OH} ;{ }^{5}$ University of Arizona, Tucson, AZ (previous); Mayo Clinic, Scottsdale, AZ (current); ${ }^{6}$ Department of Hematology and HCT, City of Hope, Duarte, CA; ${ }^{7}$ University of Rochester, James P. Wilmot Cancer Center, Rochester, NY; ${ }^{8}$ Division of Hematology/Oncology, UNC School of Medicine, Chapel Hill, NC; ${ }^{9}$ Division of Oncology, Department of Medicine, Washington University School of Medicine, Saint Louis, MO; ${ }^{10}$ Division of Hematology, Ohio State University, Columbus, $\mathrm{OH} ;{ }^{11}$ Division of Hematology \& Oncology, Medical College of Wisconsin, Milwaukee, WI; ${ }^{12}$ Wilmot Cancer Institute, University of Rochester, Rochester, NY; ${ }^{13}$ University of Michigan, Ann Arbor, MI; ${ }^{14}$ Fox Chase Cancer Center/Temple University School of Medicine, Philadelphia, PA; ${ }^{15}$ Lombardi Comprehensive Cancer Center, Georgetown University Hospital, Washington, DC; ${ }^{16}$ University of Chicago, Chicago, IL; ${ }^{17}$ Adaptive Biotechnologies Corp, South San Francisco, CA; ${ }^{18}$ Department of Hematology/Oncology, Weill Cornell Medical College, New York, NY; ${ }^{19}$ University of Wisconsin, Madison

This is the author manuscript accepted for publication and has undergone full peer review but has not been through the copyediting, typesetting, pagination and proofreading process, which may lead to differences between this version and the Version of Record. Please cite this article as doi: $10.1111 /$ bjh.14480

This article is protected by copyright. All rights reserved 
(previous); Washington University School of Medicine in St. Louis, St. Louis, MO (current); ${ }^{20}$ University of Rochester, Rochester, NY

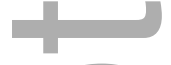 \\ Corresponding Author: Robert Chen \\ Department: Department of Hematology and Hematopoietic Cell Transplantation \\ City of Hope \\ Address: 1500 E. Duarte Rd, Duarte, CA 91010 \\ Email: rchen@coh.org \\ Phone: 6262564673 ext 84762 \\ Fax: 6263018116
}

\title{
Running Title: R-bendamustine versus R-hyperCVAD for MCL
}

Received 12 August 2016, accepted 11 October 2016S u m mary

Aggressive induction chemotherapy followed by autologous haematopoietic stem cell transplant (auto-HCT) is effective for younger patients with mantle cell lymphoma (MCL). However, the optimal induction regimen is widely debated. The Southwesterm Oncology Group S1106 trial was designed to assess rituximab plushyperCVAD/MTX/ARAC (hyperfractionated cyclophosphamide, vincristine, doxorubicin and dexamethasone, alternating with high dose cytarabine and methotrexate) $(\mathrm{RH})$ versus rituximab plus bendamustine $(\mathrm{RB})$ in a randomized phase II trial to select a pre-transplant induction regimen for future development. Patients had previously untreated stage III, IV, or bulky stage $\| \mathrm{MCL}$ and received either 4 cycles of $\mathrm{RH}$ or 6 cycles of $\mathrm{RB}$, followed by autoHCT. Fifty-three of a planned 160 patients were accrued; an unacceptably high mobilization failure rate (29\%) on the $\mathrm{RH}$ arm prompted premature study closure. The estimated 2-year progression-free survival (PFS) was $81 \%$ vs. $82 \%$ and overall survival (OS) was $87 \%$ vs. $88 \%$ for $\mathrm{RB}$ and $\mathrm{RH}$, respectively. $\mathrm{RH}$ is not an ideal platform for 
future multi-centre transplant trials in MCL. RB achieved a 2-year PFS of $81 \%$ and a $78 \%$ MRD negative rate. Premature closure of the study limited the sample size and the precision of PFS estimates and MRD rates. However, RB can achieve a deep remission and could be a platform for future trials in MCL.

\section{Keywords:}

mantle cell, bendamustine, Auto-HCT, MRD, hyperCVAD

In troduction

Mantle cell lymphoma (MCL) is an aggressive B cell non-Hodgkin lymphoma that is considered incurable with conventional therapy. It represents about $4 \%$ of lymphomas in the US (The Non Hodgkin's Lymphoma Classification Project 1997). For younger patients who are fit, the therapeutic strategy has been to use aggressive induction chemotherapy followed by autologous haematopoietic stem cell transplantation (AutoHCT). The benefit of consolidative Auto-HCT was demonstrated by the European MCL network in a trial that compared consolidative Auto-HCT to maintenance interferon and showed improved progression-free survival (PFS) in the Auto-HCT arm (median of 39 months versus 17 months $[P=0.0108]$ )(Dreyling, et al 2005). Although there are a variety of upfront regimens, the optimal induction chemotherapy has not been defined. The goal of induction chemotherapy has been to achieve a high rate of complete response $(C R)$ while minimizing toxicities.

The R-hyperCVAD $(\mathrm{RH})$ regimen pioneered at MD Anderson Cancer Center contains rituximab with hyperfractionated cyclophosphamide, vincristine, doxorubicin and dexamethasone, alternating with high dose cytarabine and methotrexate for 6-8 cycles. It yields a high CR rate of $87 \%$ and overall response rate (ORR) of $97 \%$ in single institution settings (Romaguera, et al 2005). It has also been tested in the multicentre cooperative group setting by the Southwesterm Oncology Group (SWOG) S0213 trial in patients with previously untreated $\mathrm{MCL}$, resulting in a CR of $55 \%$, ORR of $86 \%$ and 3 year PFS of $66 \%$ (Bernstein, et al 2013). Single centre studies have demonstrated the feasibility of Auto-HCT after abbreviated courses of RH (Till, et al 2008).

Rummel et al (2005) studied the combination of rituximab plus bendamustine (RB) in patients with untreated MCL and showed an ORR of $75 \%$ and $C R$ of $50 \%$, with minor haematological toxicities. This regimen was then compared to RCHOP (rituximab, cyclophosphamide, doxorubicin, vincristine, prednisolone) in a large randomized noninferiority trial in Europe, which showed similar ORR rates (93\% vs. 91\%) and CR rates 
(40\% vs. 30\%) to RCHOP but improved median PFS rates (35 months vs. 22 months), fewer haematological toxicities (30\% vs. 68\%), and fewer infections (37\% vs.

$50 \%)($ Rummel, et al 2013). These results were confirmed by the BRIGHT study, which showed that RB is non-inferior to RCHOP in terms of $\mathrm{CR}$ and had less neutropenia (Flinn, et al 2014). However, those trials did not include patients consolidated with AutoHCT.

S1106 was designed to assess $\mathrm{RH}$ and $\mathrm{RB}$ to select an induction regimen followed by Auto-HCT consolidation as a platform for development in future trials. The hypothesis was that either $\mathrm{RH}$ or RB would yield a higher 2-year PFS rate, have fewer toxicities, and allow sufficient stem cell mobilization for Auto-HCT consolidation. Besides using radiographic imaging to assess for response, we can further characterize response status by the use of minimal residual disease (MRD) monitoring. Deep remission as defined by MRD status post-induction appears to be a powerful predictor of outcome in both single arm (Liu et al 2012) and randomized settings (Pott et al 2010). In this study, we use next generation sequencing (NGS) to evaluate MRD status as an important biological correlative.

Me thods

Patients

Patients in this study (\#NCT01412879) were required to have untreated stage III, IV, or bulky stage II MCL. Cyclin D1 (CCND1) positivity was confirmed by immunohistochemistry (IHC) or fluorescence in situ hybridization (FISH), and all pathological material underwent central histological review through SWOG. Other inclusion criteria were age $\geq 18 \leq 65$ years, adequate organ function, no prior treatment for MCL, bidimensionally measurable disease $>1.5 \mathrm{~cm}$ by computed tomography $(\mathrm{CT})$, fluorodeoxyglucose (FDG)-avid disease by positron emission tomography (PET), and a Zubrod performance status score of 0 - 2.

Study Design and Treatment

This randomized phase II study was conducted by SWOG through the National Clinical Trials Network (NCTN) adult groups (SWOG/ECOG-ACRIN/Alliance)

mechanism. Patients were recruited from January 4, 2012 through June 21, 2013. The study was approved by the Institutional Review Board at each study site, and written informed consent was obtained from all patients prior to any study-specific procedures, per the Declaration of Helsinki. The study was approved and sponsored by the Cancer Therapy Evaluation Program (CTEP). Patients were randomized to 4 cycles of $\mathrm{RH}$ or 6 
cycles of RB (Fig 1). Randomization was stratified on the MCL International Prognostic Index (MIPI) score (Hoster, et al 2008). The dose of RH and RB were previously described (Bernstein et al 2013; Rummel et al 2005)(Table I). Only patients achieving $\mathrm{CR}$ or PR at restaging were eligible to proceed to Auto-HCT consolidation. Patients in the $\mathrm{RH}$ arm underwent chemotherapy-based stem cell mobilization after cycle 3 with granulocyte colony-stimulating factor(dose/schedule per institutional standard). Patients in the RB arm underwent stem cell mobilization after cycle 6 (within 8 weeks of last dose of RB) using rituximab $\left(375 \mathrm{mg} / \mathrm{m}^{2}\right)$ plus cyclophosphamide $\left(1.5 \mathrm{mg} / \mathrm{m}^{2}\right)$. Plerixafor or a second mobilization attempt was allowed but not required per protocol. After at least 1.5 $\times 10^{6}$ CD34+ cells were collected, patients underwent a second registration and proceeded to the transplant component of planned treatment. For patients between 61 and 65 years old, each site had to select either CBV (carmustine [BCNU]/cyclophosphamide/etoposide) or BEAM (BCNU/etoposide/cytarabine/melphalan) as the sole preparative regimen (Chopra, et al 1993, Reece, et al 1994) for that site. For patients aged less than 61 years, the sites had to select either CBV, BEAM, or total body irradiation/cyclophosphamide/etoposide (Nademanee, et al 1995) as the sole preparative regimen.

Minimal residual disease (MRD) was assessed at baseline and post-induction from peripheral blood. Genomic tumour DNA was extracted from FPE formalin-fixed paraffin-embedded tissue or bone marrow aspirate mononuclear cells. Polymerase chain reaction (PCR) amplification of IGH VDJ, IGH DJ, and IGK regions was performed followed by next-generation sequencing to determine the tumour clonotype(s) (Adaptive Biotechnologies Corp, South San Francisco, CA)(Faham, et al 2012). DNA from peripheral blood mononuclear cells (PBMC) and plasma was amplified and sequenced to determine lymphoma molecules per million diploid genomes. If either the PBMC or the plasma sample yielded lymphoma clones, then the patient was deemed to be MRD positive.

Study Assessments

Baseline evaluations included documentation of disease-related signs and symptoms, a physical examination, bone marrow biopsy and radiographic studies, including CT of the neck, chest, abdomen and pelvis, and a PET scan. The best clinical response was determined by investigators according to the Revised Response Criteria for Malignant Lymphoma (Cheson, et al 2007). In the $\mathrm{RH}$ arm, response was assessed by CT scan after cycles 2 and 4 , and a PET scan was done after cycle 4 but prior to 
Auto-HCT. In the RB arm, CT scans were specified after cycles 4 and 6, and a PET scan was done after cycle 6 but prior to Auto-HCT. After Auto-HCT and after removal from study treatment, long-term follow up assessments (including survival and disease status information) were performed every 3 months for 1 year, every 6 months for 2 year, then annually thereafter until either patient death or 8 years after registration. Patients who discontinued study treatment with stable disease or better had CT scans every 6 months for 3 years and annually thereafter until disease progression.

Safety monitoring included the recording of adverse events and physical examination findings, vital signs, and routine haematology and serum chemistries.

Adverse events were graded using the National Cancer Institute's Common Terminology Criteria for Adverse Events, version 4 (http://evs.nci.nih.gov/ftp1/CTCAE/CTCAE_4.03_2010-06-

14_QuickReference_8.5x11.pdf).

Statistical Analysis

The primary objective of this Phase II design was to select a regimen for further development, and the primary endpoint is estimated 2-year PFS. Recent SWOG experience in this patient population in S0213 (Bernstein, et al 2013) and S0601 (Till, et al 2016) suggested a historical 2-year PFS of $68 \%$. In order to proceed with development, the observed 2-year PFS on the corresponding arm must be at least $75 \%$. Under the original design and sample size, this threshold corresponds not only to a 1sided 0.05 level test of the null hypothesis that the true 2-year PFS is $68 \%$, but also approximates the outcomes observed with the transplant regimen of the Nordic Lymphoma Group (Geisler, et al 2008).

Interim analyses of stem cell collection data on both treatment arms was conducted by the statistical centre. Either arm would be considered unacceptably toxic if the true stem cell collection failure rate exceeded 10\%. If, among the first 20 eligible patients on either arm, more than 4 (20\%) were unable to receive transplant because of inadequate stem cell collection, then protocol accrual would be held and consideration would be given to modifications or closure of that arm. These analyses correspond to 1sided 0.05 level tests of the null hypothesis that the true failure rate is $10 \%$ or less.

Secondary endpoints included ORR, CR, OS, toxicities and MRD analysis. The probability of response or a particular toxicity can be estimated to be within at worst $11 \%$ (95\% confidence interval [Cl]) with 80 patients per arm. Any adverse event with at least a $5 \%$ probability would be seen at least once ( $98 \%$ chance). This protocol also assessed 
the association of MRD status at the end of induction therapy, prior to stem cell transplant, with PFS. PFS and OS estimates (with 95\% Cl) were calculated using Kaplan-Meier methods (Kaplan and Meier 1958). To minimize selection bias, a landmark analysis at 3 months for $\mathrm{RH}$ and at 6 months for $\mathrm{RB}$ was used for comparing PFS between transplanted and non-transplanted patients. In this analysis, only patients on $\mathrm{RH}$ with at least 3 months of follow-up without progression or patients on RB with 6 months of follow-up without progression were included, and the subsequent progressionfree time after 3 months of follow-up for $\mathrm{RH}$ and after 6 months of follow-up for RB were compared. Four patients on RB who did not receive Auto-HCT were excluded from this landmark analysis, as three patients were censored and one patient progressed within the 6-months landmark time. The estimated 2-year PFS for patients on RB who achieved MRD-negative status at the end of induction was also calculated by Kaplan-Meier methods using landmark analysis at time of specimen collected for MRD testing. Comparisons of response rate and toxicity rate between treatment arms were performed using Fisher's exact test with two-sided alpha of 0.05. Data as of 10 February 2016 were included in the analyses.

\section{Results \\ Patients}

Patient characteristics are detailed in Table II. This study was closed after 53 patients were accrued: 18 in $\mathrm{RH}$ and 35 in $\mathrm{RB}$ arms ( $\mathrm{RH}$ arm closing first). One patient on $\mathrm{RH}$ who did not receive any randomized therapy due to the closure of $\mathrm{RH}$ arm for safety was excluded from all endpoint analyses. The groups were well-balanced (Table II) with the exception of more females in the $\mathrm{RH}$ group. The median age was 57 (range, 33-64) years in the RB arm and 58 (range, 44-66) years in the $\mathrm{RH}$ arm. Ninety-one per cent of the patients in the RB arm and $94 \%$ of the patients in the $\mathrm{RH}$ arm had stage IV disease. Eighty-six per cent of the patients in the RB arm and $82 \%$ of the patients in the $\mathrm{RH}$ arm had bone marrow involvement. Ninety-one per cent of the patients in the RB arm and $88 \%$ of the patients in the $\mathrm{RH}$ arm had extra-nodal disease. Lastly, $37 \%$ of the patients in the RB arm and $35 \%$ of the patients in the $\mathrm{RH}$ arm were considered intermediate/high risk by MIPI score. Approximately $20 \%$ of the patients in each arm were considered high risk as determined by a Ki67 score $>30 \%$. 


\section{Efficacy}

The ORR was $94.1 \%$ in the $\mathrm{RH}$ arm versus $82.9 \%$ in the $\mathrm{RB}$ arm $(\mathrm{p}=0.4)$. The CR rates were $40 \%$ and $35 \%$ for $\mathrm{RB}$ and $\mathrm{RH}$, respectively, and the $\mathrm{PR}$ rates were $43 \%$ and $59 \%$ for $\mathrm{RB}$ and $\mathrm{RH}$, respectively. One patient in the $\mathrm{RH}$ arm and 6 patients in the $\mathrm{RB}$ arm were not evaluable and considered to be non-responders (did not receive radiographic imaging). The median follow-up was 37 months in the $\mathrm{RH}$ arm and 33 months in the RB arm. The estimated 2-year PFS was $81 \%$ vs. $82 \%$ and estimated 2year OS was $87 \%$ vs. $88 \%$ for $\mathrm{RB}$ and $\mathrm{RH}$ respectively (Figs $2-3$ ). In the $\mathrm{RH}$ arm, 5 patients received Auto-HCT on protocol, and 4 patients received Auto-HCT off protocol (discontinued therapy early for toxicities). The 2-year PFS estimates from the landmark at 3 months of follow-up are $75 \%$ vs. $88 \%(p=0.43)$ for patients that underwent AutoHCT versus those who did not. In the RB arm, 21 patients received Auto-HCT on protocol, 2 received Auto-HCT off protocol, and 12 patients did not receive Auto-HCT. The 2-year PFS estimates from the landmark at 6 months of follow-up for patients who did not or did receive Auto-HCT was $60 \%$ vs. $81 \%(p=0.20)$.

Safety

The $\mathrm{BH}$ arm had significantly more marrow toxicity compared to the $\mathrm{RB}$ arm, with increased Grade 3 or 4 thrombocytopenia (71\% vs. 17\%) ( $<<0.001)$, anaemia (59\% vs. $8.6 \%)(p<0.001)$, neutropenia (65\% vs. $34 \%)(p=0.07)$, and febrile neutropenia ( $29 \%$ vs. 14\%) ( $p=0.26)$ (Table III). The RB arm also had fewer observed Grade 3 or 4 nonhaematological adverse events, but numbers are too small for a definitive comparison. Grade 3 or 4 non-haematological adverse events that occurred in greater than $5 \%$ of the patients on the $\mathrm{RH}$ arm were hypokalaemia (29\%), hypophosphataemia (24\%), hyperglycaemia (12\%), aspartate transaminase (AST) elevation $(5.9 \%)$, alanine transaminase (ALT) elevation (5.9\%), catheter-related infection (5.9\%), dehydration $(5.9 \%)$, diarrhoea (5.9), epistaxis (5.9\%), nausea (5.9), rash (5.9\%) and syncope (5.9\%). The sole grade 3 or 4 non-haematological adverse event that occurred in greater than $5 \%$ of patients on the RB arm was hypokalaemia (5.7\%).

Twenty-six patients did not finish the course of induction chemotherapy followed by Auto-HCT. On the RH arm, 7/17 patients could not finish induction chemotherapy, mostly because of delayed platelet recovery (Table IV). An additional 5 patients could not mobilize an adequate number of stem cells after cycle 2 of induction chemotherapy. This failure to collect an adequate number of stem cells triggered the early stopping rules, and the $\mathrm{RH}$ arm was closed. On the $\mathrm{RB}$ arm, 8/35 could not finish induction 
chemotherapy and 6 did not undergo Auto-HCT per protocol (Table IV). However, only 2 patients could not mobilize an adequate number of stem cells. The detailed reasons for stopping therapy early are listed in Table IV. The entire study was halted and closed because the $\mathrm{RH}$ arm encountered the early stopping rule. A result was a smaller sample size of the whole study, which limited the precisions around our PFS estimates.

\section{$M R D$ analysis}

This study also analysed MRD status post-induction therapy via NGS. Twentyseven patients consented to the optional MRD analysis, with 12 paired specimens (preand post-induction therapy, 2 in $\mathrm{RH}$ and 10 in $\mathrm{RB}$ ). In the $\mathrm{RH}$ arm, 2 patients were MRD positive at baseline; both became MRD negative at the end of induction. In the RB arm, 1 patient was MRD negative at baseline, and 9 patients were MRD positive. Seven out of nine patients converted to MRD negativity at the end of induction (MRD negative rate of $78 \%$ ). Interestingly, the remaining 2 patients that were MRD positive at end of induction had discordant MRD results between the PBMC and plasma samples. For both patients, MRD analysis of the PBMC sample yielded low positive MRD at the end of induction (One patient had a 6570-fold reduction and another patient had 22000 -fold reduction in the lymphoma clonotype per million diploid genome). However, the MRD analysis of the plasma sample yielded an MRD negative result. These two patients remain alive and in remission. One patient on $\mathrm{RB}$ was $\mathrm{MRD}$ negative at baseline and remained MRD negative at the end of induction. This patient eventually developed progressive disease.

There were 3 additional patients that were missing the baseline MRD analysis specimens (not included in the 12 paired samples), but were MRD negative at the end of induction. One of the 10 patients who was MRD negative at end of induction died approximately 3 months after receiving protocol Auto-HCT, and the rest remain in remission, with a 2-year landmark PFS of $90 \%$ (95\% Cl: $47.3 \%, 98.5 \%)$.

\section{Discussion}

In this randomized multicentre phase II prospective trial, we showed that both $\mathrm{R}$ hyperCVAD $(\mathrm{RH})$ and $\mathrm{R}$-bendamustine $(\mathrm{RB})$ are active induction regimens with high overall response rates. While the observed complete remission rates were lower than historical controls, this may reflect that functional imaging was not mandatory at the first restaging, and several patients stopped the study early for toxicity and thus did not finish the induction therapy or undergo the post induction FDG scan (Bernstein, et al 2013, Romaguera, et al 2005). There were significant differences in toxicity, with the $\mathrm{RH}$ arm 
displaying significantly more grade 3 and 4 anaemia, neutropenia and thrombocytopenia. Furthermore, almost one-third of patients on the $\mathrm{RH}$ arm failed to mobilize even $1.5 \times 10^{6}$ stem cells, prompting protocol-specified early termination of accrual to the $\mathrm{RH}$ arm. Historically, R-hyperCVAD was developed as an 8-cycle regimen without stem cell transplantation consolidation. Single institution studies shortened the $\mathrm{RH}$ regimen to 4 cycles, followed by Auto-HCT, which appeared feasible (Till, et al 2008). However, our prospective multicentre trial clearly shows that abbreviated $\mathrm{RH}$ followed by Auto-HCT is not a viable induction strategy when stem cell transplantation is planned. In contrast, the toxicity profile of the RB arm was similar to prior trials (Rummel et al 2005; Rummel et al 2013; Flinn et al 2014) and well tolerated. However, $40 \%$ of the patients did not proceed to Auto-HCT as expected (6\% for mobilization failure, $6 \%$ for progressive disease, $6 \%$ for other reasons, $9 \%$ for toxicity and $14 \%$ for patient choice or insurance denial). Although the total number of 14 appears to be high, the numbers were low (1-2) for each individual reason given, with the exception of patient choice. Because of the small sample size from early trial closure, the true significance of this data is unclear. The most common reason for patients not proceeding to Auto-HCT was patient choice. It is unclear as to why patients chose not to proceed with Auto-HCT. One possibility could be the transition from induction treatment physician to a different transplant physician as many centres have a separate transplantation team. It also may simply represent the challenges of conducting a randomized multi-step trial involving Auto-HCT in the cooperative group setting. However, given that the ORR rate appears similar in both $\mathrm{RH}$ and $\mathrm{RB}$ arms, but $\mathrm{RH}$ has more haematological toxicity and stem cell mobilization insufficiency, our study supports RB as a more favourable induction backbone than $\mathrm{RH}$ for MCL.

The trial's primary endpoint was to target a 2-year PFS estimate of $75 \%$. The 2year PFS estimates of both $\mathrm{RH}$ and $\mathrm{RB}$ arms were similar at $82 \%$ and $81 \%$, respectively. Because the study closed prematurely, the precision around the PFS estimates is limited. However, the 2-year PFS estimate of $81 \%$ is higher than the planned $75 \%$. The 2 -year OS was also similar in both arms at $87 \%$ vs. $88 \%$, suggesting that both induction regimens followed by auto-HCT may be similar in terms of efficacy, although the small sample sizes limit inference. In the $\mathrm{RH}$ arm, 5 patients received Auto-HCT on protocol, and 4 patients received Auto-HCT off protocol. The 2-year landmark PFS estimates are $75 \%$ vs. $88 \%$ for patients that underwent Auto-HCT versus those who did not, suggesting that Auto-HCT may not substantially affect 2-year PFS. However, this 
analysis is limited by the small sample size. In the RB arm, 21 patients received AutoHCT on protocol, 2 received Auto-HCT off protocol and 12 patients did not receive AutoHCT. Although the 2-year landmark PFS estimates were appearing more promising for patients who received Auto-HCT ( $81 \%$ vs. $60 \%)(p=0.20)$, this difference does not meet statistical significance.

Minimal residual disease (MRD) appears to be a potent predictor of outcome, and was included as an optional correlative study. Historically, real-time quantitative PCR analysis of rearranged immunoglobulin heavy chain $(I G H)$ genes and multicolour flow cytometry have been used for MRD assessment in MCL (Pott 2011). Studies using these methods show that MRD negative status post therapy can predict for long term remission (Bottcher, et al 2008, Pott, et al 2010). However, these approaches have a number of limitations, including low sensitivity, failure of marker identification, and requirement of patient-specific strategies, which is more cumbersome. NGSuses locusspecific primer sets of $I G H$ and IGK regions to amplify and sequence cancer-derived clones (Faham, et al 2012). These cancer-derived sequences are then used as targets that assess for the presence of MRD in follow-up samples. In our study, we evaluated MRD status with NGS technology. Twenty-seven patients consented to MRD testing, with 12 paired samples of both pre- and post-induction material (peripheral blood). Despite this limited sample size, both $\mathrm{RH}$ and $\mathrm{RB}$ achieved a relatively high rate of MRD negative status. Patients with conversion from MRD positive to MRD negative status post-induction had excellent outcomes with a 2-year landmark PFS of $90 \%$; three patients did not proceed to Auto-HCT. We also observed discordance between MRD results from PBMC and plasma samples. It is unclear whether MRD results from PBMC or plasma samples are more sensitive or specific. Two patients were low-level MRD positive (low number of molecules) by PMBC and negative by plasma at the end of induction. These two patients had a dramatic reduction in their lymphoma clonotype molecules (6570-fold for one and 22000-fold for another) and still remain in remission. The clinical significance of low level MRD positivity is unknown at this time and will need to be evaluated in future studies. Although these provocative findings require confirmation in a larger study, our data suggest that NGS-based MRD-negative status is feasible after RB and could become a new endpoint for future studies and allow for more rapid evaluation of new treatment strategies.

The Nordic Lymphoma Group introduced the concept of adding cytarabine to the induction regimen of MCL. In their single arm prospective study, the addition of 
cytarabine to induction followed by Auto-HCT resulted in a 6-year OS of 70\% and PFS of 66\% (Geisler, et al 2008). Of the 160 patients, 145 (91\%) were able to collect stem cells and proceed to Auto-HCT. Although this was not a randomized trial, this argues for inclusion of cytarabine in the induction regimen. A recent publication from the European Mantle Cell Lymphoma Network (Hermine, et al 2016) compared 6 cycles of R-CHOP to 6 cycles of alternating R-CHOP and R-DHAP (rituximab, dexamethasone, high-dose cytarabine, cisplatin) as induction regimen and suggested that the addition of cytarabine to induction chemotherapy improves outcomes post Auto-HCT. This study showed an improved 5-year PFS in the cytarabine group (65\% vs. 44\%). It may appear that the result from this study is contradictory to our study, as our conclusion was to avoid $\mathrm{RH}$, a cytarabine-containing regimen. However, there are a number of similarities in both studies, and the difference lies in the interpretation of the data. For example: 1) the confirmed CR rate of R-CHOP/R-DHAP was $38 \%$, which is similar to the confirmed CR of RB (40\%); 2) the MRD negative rate from R-CHOP/R-DHAP was $79 \%$, which is similar to the MRD negative rate from RB (78\%); 3) the 2-year PFS and OS from RCHOP/R-DHAP appear similar to the 2-year PFS and OS from RB. These findings would suggest that, although the addition of cytarabine into the induction therapy is advantageous to R-CHOP, it may not be necessary when compared to RB. Finally, the stem cell collection failure rate was much higher in the R-CHOP/R-DHAP arm as compared to the R-CHOP arm (34\% vs. $16 \%$ ). Although the Nordic Lymphoma Group did not have problems with stem cell collection with the inclusion of cytarabine, it appears that the inclusion of cytarabine and cisplatin (R-DHAP) contributes to stem cell mobilization failure.

A variety of novel therapies have been tested in recent years for relapsed/refractory $\mathrm{MCL}$. Bortezomib, a proteasome inhibitor, has demonstrated an ORR of $33 \%$ and a CR rate of $8 \%$ in this setting (Fisher, et al 2006). Patients treated with the immunomodulatory agent lenalidomide were shown in a phase II trial to have an ORR of $35 \%$ and a CR rate of $12 \%$ (Zinzani, et al 2013). Ibrutinib, a Bruton tyrosine kinase inhibitor (Wang, et al 2013), has shown an ORR of $68 \%$ and a CR of $21 \%$. These agents are now being incorporated into the induction setting as combination therapies. Early results from an induction trial of lenalidomide plus rituximab showed an ORR of $92 \%$, CR of $64 \%$ and a 2 -year PFS of $85 \%$ (Ruan, et al 2015). The ORR and PFS were similar to those in our study and other induction regimens. However, this was a single arm study that excluded patients with high-risk MIPI score (unless there were contraindications to 
chemotherapy) and required patients to be treated for at least 36 cycles of therapy unless there was excess toxicity or progressive disease. These results need to be confirmed in a larger randomized trial with longer follow-up time. It is possible that the addition of novel therapies into induction regimens could result in greater $C R$ rates, higher MRD negative responses post-induction, and longer PFS. Although the current standard treatment for younger patients is consolidation in first CR post-induction with Auto-HCT, it is possible that inclusion of novel therapies into induction or as consolidation may change this standard in the future. The current European Network younger trial and future US intergroup trials will attempt to answer this provocative question.

Overall, significant progress has been made in understanding the molecular biology of MCL, and that has translated into improved outcome. The median OS of patients with MCL has been extended from 2.7 years to 4.8 years from the time period of 1975-1996 to 1996-2004 (Herrmann, et al 2009). Since then, other trials have further shown an improvement in OS. The Nordic group used an induction regimen of R-maxi$\mathrm{CHOP}$ alternating with cytarabine followed by Auto-HCT, showing a median event-free survival (EFS) of 7.4 years and median OS of greater than 10 years (Geisler, et al 2012). Many novel agents have been discovered and will certainly extend the median OS even further.

In conclusion, this SWOG-led NCTN trial showed that RH was difficult to incorporate in a multicentre setting because of toxicity and inadequate stem cell mobilization, and is thus not ideal for future development in this setting. In contrast, RB had high response rates, a 78\% MRD negativity rate, and was generally well tolerated with successful stem cell mobilization. In addition, patients achieving MRD negative status after induction had excellent outcomes reflected by a 2-year landmark PFS of $90 \%$. We recognize that $40 \%$ of the patients on the RB arm did not proceed to Auto-HCT per protocol and that the sample size was small. Thus, the results of this trial need to be confirmed. The current Eastern Cooperative Oncology Group-led NCTN trial (NCT 01415752) using RB with or without bortezomib, followed by rituximab with or without lenalidomide, could support our observation. Upon confirmation, future trials should focus on MRD analysis as primary endpoints for induction trials, as well as the role of Auto-HCT consolidation therapy in patients who are MRD negative post-induction therapy.

This article is protected by copyright. All rights reserved 
Acknowledgments This work was supported in part by National Institute of Health, National Cancer Institute grants CA180888, CA180819, CA180821, CA180820, CA189957, CA189821, CA189972, CA189953, CA180846, CA180835, CA189808, CA11083, CA46368, CA46136 CA76462, and in part by Sequenta, Inc. (Adaptive Biotechnologies).

\section{Authorship}

Designed research: RC, SJF, BC, SB, LC, JF, MF, RF, BK, ML, TS, JW.

Performed research: RC, SJF, KB, PB, AC, JF, BK, HL, JL, TS, JW.

Collected data: RC, KB, TF, HL, JL.,L.R, JW.

Analysed and interpreted data: RC, SJF, PB, SB, AC, JF, MF, RF, TF, BK, HL, JL, TP. LR, SS, JW.

Performed statistical analysis: HL.

Wrote manuscript: RC, SJF, PB, SB, BC, JF, BK, HL, SS, TS.

Reviewed manuscript: All authors.

\section{Disclosures}

No completing financial interests: SJF, KB, PB, SB, BC, LC, RF, SK, HL, ML, TP, LR, SS, JW.

Membership on another entity's Board of Directors or its advisory committees (whether for profit or not for profit): R.C. serves on Advisory Boards of Merck, Seattle Genetics, and Genentech, and serves on Speaker Bureau for Seattle Genetics, Millennium, and Genentech. J.F. serves on Bayer Data and Safety Monitoring Board; T.S. serves on Advisory Boards of Novartis, Spectrum, Seattle Genetics, and Merck; as consultant for Teva: J.L.; Consulting with Teva and Genentech: B.K.; Consulting with Celgene, Seattle Genetics, Pharmacyclics and Sanofi: T.F.; Research funding through his University from Millennium, Otsuka, GSK, Novartis, and BMS: T.S.; Speakers' Bureau for Celgene: A.C.; M.F. is Stockholder, Adaptive Biotechnolgies.

\section{References}


Bernstein, S.H., Epner, E., Unger, J.M., LeBlanc, M., Cebula, E., Burack, R., Rimsza, L., Miller, T.P. \& Fisher, R.I. (2013) A phase II multicenter trial of hyperCVAD MTX/Ara-C and rituximab in patients with previously untreated mantle cell lymphoma; SWOG 0213. Annals of Oncology, 24, 1587-1593.

Bottcher, S., Ritgen, M., Buske, S., Gesk, S., Klapper, W., Hoster, E., Hiddemann, W., Unterhalt, M., Dreyling, M., Siebert, R., Kneba, M. \& Pott, C. (2008) Minimal residual disease detection in mantle cell lymphoma: methods and significance of four-color flow cytometry compared to consensus IGH-polymerase chain reaction at initial staging and for follow-up examinations. Haematologica, 93, 551-559.

Cheson, B.D., Pfistner, B., Juweid, M.E., Gascoyne, R.D., Specht, L., Horning, S.J., Coiffier, B., Fisher, R.I., Hagenbeek, A., Zucca, E., Rosen, S.T., Stroobants, S., Lister, T.A., Hoppe, R.T., Dreyling, M., Tobinai, K., Vose, J.M., Connors, J.M., Federico, M. \& Diehl, V. (2007) Revised Response Criteria for Malignant Lymphoma. Journal of Clinical Oncology, 25, 579-586.

Chopra, R., McMillan, A.K., Linch, D.C., Yuklea, S., Taghipour, G., Pearce, R., Patterson, K.G. \& Goldstone, A.H. (1993) The Place of High-Dose BEAM Therapy and Autologous Bone Marrow Transplantation in Poor-Risk Hodgkin's Disease. A Single-Center Eight-Year Study of 155 Patients. Blood, 81, 11371145.

Dreyling, M., Lenz, G., Hoster, E., Van Hoof, A., Gisselbrecht, C., Schmits, R., Metzner, B., Truemper, L., Reiser, M., Steinhauer, H., Boiron, J.M., Boogaerts, M.A., Aldaoud, A., Silingardi, V., Kluin-Nelemans, H.C., Hasford, J., Parwaresch, R., Unterhalt, M. \& Hiddemann, W. (2005) Early consolidation by myeloablative radiochemotherapy followed by autologous stem cell transplantation in first remission significantly prolongs progression-free survival in mantle-cell lymphoma: results of a prospective randomized trial of the European MCL Network. Blood, 105, 2677-2684.

Faham, M., Zheng, J., Moorhead, M., Carlton, V.E., Stow, P., Coustan-Smith, E., Pui, C.H. \& Campana, D. (2012) Deep-sequencing approach for minimal residual disease detection in acute lymphoblastic leukemia. Blood, 120, 5173-5180.

Fisher, R.I., Bernstein, S.H., Kahl, B.S., Djulbegovic, B., Robertson, M.J., de Vos, S., Epner, E., Krishnan, A., Leonard, J.P., Lonial, S., Stadtmauer, E.A., O'Connor, O.A., Shi, H.L., Boral, A.L. \& Goy, A. (2006) Multicenter phase II study of 
bortezomib in patients with relapsed or refractory mantle cell lymphoma. Journal of Clinical Oncology, 24, 4867-4874.

Flinn, I.W., van der Jagt, R., Kahl, B.S., Wood, P., Hawkins, T.E., Macdonald, D., Hertzberg, M., Kwan, Y.L., Simpson, D., Craig, M., Kolibaba, K., Issa, S., Clementi, R., Hallman, D.M., Munteanu, M., Chen, L. \& Burke, J.M. (2014) Randomized trial of bendamustine-rituximab or R-CHOP/R-CVP in first-line treatment of indolent NHL or MCL: the BRIGHT study. Blood, 123, 2944-2952.

Geisler, C.H., Kolstad, A., Laurell, A., Andersen, N.S., Pedersen, L.B., Jerkeman, M., Eriksson, M., Nordstrom, M., Kimby, E., Boesen, A.M., Kuittinen, O., Lauritzsen, G.F., Nilsson-Ehle, H., Ralfkiaer, E., Akerman, M., Ehinger, M., Sundstrom, C., Langholm, R., Delabie, J., Karjalainen-Lindsberg, M.L., Brown, P. \& Elonen, E. (2008) Long-term progression-free survival of mantle cell lymphoma after intensive front-line immunochemotherapy with in vivo-purged stem cell rescue: a nonrandomized phase 2 multicenter study by the Nordic Lymphoma Group.

Blood, 112, 2687-2693.

Geisler, C.H., Kolstad, A., Laurell, A., Jerkeman, M., Raty, R., Andersen, N.S., Pedersen, L.B., Eriksson, M., Nordstrom, M., Kimby, E., Bentzen, H., Kuittinen, O., Lauritzsen, G.F., Nilsson-Ehle, H., Ralfkiaer, E., Ehinger, M., Sundstrom, C., Delabie, J., Karjalainen-Lindsberg, M.L., Brown, P. \& Elonen, E. (2012) Nordic MCL2 trial update: six-year follow-up after intensive immunochemotherapy for untreated mantle cell lymphoma followed by BEAM or BEAC + autologous stemcell support: still very long survival but late relapses do occur. British Journal of Haematology, 158, 355-362.

Hermine, O., Hoster, E., Walewski, J., Bosly, A., Stilgenbauer, S., Thieblemont, C., Szymczyk, M., Bouabdallah, R., Kneba, M., Hallek, M., Salles, G., Feugier, P., Ribrag, V., Birkmann, J., Forstpointner, R., Haioun, C., Hanel, M., Casasnovas, R.O., Finke, J., Peter, N., Bouabdallah, K., Sebban, C., Fischer, T., Duhrsen, U., Metzner, B., Maschmeyer, G., Kanz, L., Schmidt, C., Delarue, R., Brousse, N., Klapper, W., Macintyre, E., Delfau-Larue, M.H., Pott, C., Hiddemann, W., Unterhalt, M. \& Dreyling, M. (2016) Addition of high-dose cytarabine to immunochemotherapy before autologous stem-cell transplantation in patients aged 65 years or younger with mantle cell lymphoma (MCL Younger): a randomised, open-label, phase 3 trial of the European Mantle Cell Lymphoma Network. Lancet, 388, 565-575. 
Herrmann, A., Hoster, E., Zwingers, T., Brittinger, G., Engelhard, M., Meusers, P., Reiser, M., Forstpointner, R., Metzner, B., Peter, N., Wormann, B., Trumper, L., Pfreundschuh, M., Einsele, H., Hiddemann, W., Unterhalt, M. \& Dreyling, M. (2009) Improvement of Overall Survival in Advanced Stage Mantle Cell Lymphoma. Journal of Clinical Oncology, 27, 511-518.

Hoster, E., Dreyling, M., Klapper, W., Gisselbrecht, C., van Hoof, A., Kluin-Nelemans, H.C., Pfreundschuh, M., Reiser, M., Metzner, B., Einsele, H., Peter, N., Jung, W., Wörmann, B., Ludwig, W.-D., Dührsen, U., Eimermacher, H., Wandt, H., Hasford, J., Hiddemann, W. \& Unterhalt, M. (2008) A new prognostic index (MIPI) for patients with advanced-stage mantle cell lymphoma. Blood, 111, 558-565.

Kaplan, G. \& Meier, P. (1958) Non-parametric estimations from incomplete observations. J Am Stat Assoc, 53, 457-481.

Liu, H., Johnson, J.L., Koval, G., Malnassy, G., Sher, D., Damon, L.E., Hsi E.D., Bucci, D.M., Linker, C.A., Cheson, B.D., Stock, W. (2012) Detection of minimal residual disease following induction immunochemotherapy predicts progression free survival in mantle cell lymphoma: final results of CALGB 59909. Haematologica, 97, 579-585.

Nademanee,A., O'Donnell, M.R., Snyder, D.S., Schmidt, G.M., Parker, P.M., Stein, A.S., Smith, E.P., Molina, A., Stepan, D.E., Somlo, G. \& . (1995) High-Dose Chemotherapy With or Without Total Body Irradiation Followed by Autologous Bone Marrow and/or Peripheral Blood Stem Cell Transplantation for Patients with Relapsed and Refractory Hodgkin's Disease: Results in 85 Patients with Analysis of Prognos. Blood, 85, 1381-1390.

Pott, C. (2011) Minimal residual disease detection in mantle cell lymphoma: technical aspects and clinical relevance. Seminars in Hematology, 48, 172-184.

Pott, C., Hoster, E., Delfau-Larue, M.H., Beldjord, K., Bottcher, S., Asnafi, V., Plonquet, A., Siebert, R., Callet-Bauchu, E., Andersen, N., van Dongen, J.J., Klapper, W., Berger, F., Ribrag, V., van Hoof, A.L., Trneny, M., Walewski, J., Dreger, P., Unterhalt, M., Hiddemann, W., Kneba, M., Kluin-Nelemans, H.C., Hermine, O., Macintyre, E. \& Dreyling, M. (2010) Molecular remission is an independent predictor of clinical outcome in patients with mantle cell lymphoma after combined immunochemotherapy: a European MCL intergroup study. Blood, 115, 3215-3223.

This article is protected by copyright. All rights reserved 
Reece, D.E., Connors, J.M., Spinelli, J.J., Barnett, M.J., Fairey, R.N., Klingemann, H.G., Nantel, S.H., O'Reilly, S., Shepherd, J.D., Sutherland, H.J. (1994) Intensive therapy with cyclophosphamide, carmustine, etoposide +/- cisplatin, and autologous bone marrow transplantation for Hodgkin's disease in first relapse after combination chemotherapy. Blood, 83, 1193-1199.

Romaguera, J.E., Fayad, L., Rodriguez, M.A., Broglio, K.R., Hagemeister, F.B., Pro, B., McLaughlin, P., Younes, A., Samaniego, F., Goy, A., Sarris, A.H., Dang, N.H., Wang, M., Beasley, V., Medeiros, L.J., Katz, R.L., Gagneja, H., Samuels, B.I., Smith, T.L. \& Cabanillas, F.F. (2005) High rate of durable remissions after treatment of newly diagnosed aggressive mantle-cell lymphoma with rituximab plus hyper-CVAD alternating with rituximab plus high-dose methotrexate and cytarabine. Journal of Clinical Oncology, 23, 7013-7023.

Ruan, J., Martin, P., Shah, B., Schuster, S.J., Smith, S.M., Furman, R.R., Christos, P., Rodriguez, A., Svoboda, J., Lewis, J., Katz, O., Coleman, M. \& Leonard, J.P. (2015) Lenalidomide plus Rituximab as Initial Treatment for Mantle-Cell Lymphoma. New England Journal of Medicine, 373, 1835-1844.

Rummel, M.J., Al-Batran, S.E., Kim, S.Z., Welslau, M., Hecker, R., Kofahl-Krause, D., Josten, K.M., Durk, H., Rost, A., Neise, M., von Grunhagen, U., Chow, K.U., Hansmann, M.L., Hoelzer, D. \& Mitrou, P.S. (2005) Bendamustine plus rituximab is effective and has a favorable toxicity profile in the treatment of mantle cell and low-grade non-Hodgkin's lymphoma. Journal of Clinical Oncology, 23, 33833389.

Rummel, M.J., Niederle, N., Maschmeyer, G., Banat, G.A., von Grunhagen, U., Losem, C., Kofahl-Krause, D., Heil, G., Welslau, M., Balser, C., Kaiser, U., Weidmann, E., Durk, H., Ballo, H., Stauch, M., Roller, F., Barth, J., Hoelzer, D., Hinke, A. \& Brugger, W. (2013) Bendamustine plus rituximab versus CHOP plus rituximab as first-line treatment for patients with indolent and mantle-cell lymphomas: an openlabel, multicentre, randomised, phase 3 non-inferiority trial. Lancet, 381, 12031210.

The Non Hodgkin's Lymphoma Classification Project (1997) A clinical evaluation of the International Lymphoma Study Group classification of non-Hodgkin's lymphoma. Blood, 89, 3909-3918.

Till, B.G., Gooley, T.A., Crawford, N., Gopal, A.K., Maloney, D.G., Petersdorf, S.H., Pagel, J.M., Holmberg, L., Bensinger, W. \& Press, O.W. (2008) Effect of 
remission status and induction chemotherapy regimen on outcome of autologous stem cell transplantation for mantle cell lymphoma. Leukemia and Lymphoma, 49, 1062-1073.

Till, B.G., Li, H., Bernstein, S.H., Fisher, R.I., Burack, W.R., Rimsza, L.M., Floyd, J.D., DaSilva, M.A., Moore, D.F., Jr., Pozdnyakova, O., Smith, S.M., LeBlanc, M. \& Friedberg, J.W. (2016) Phase II trial of R-CHOP plus bortezomib induction therapy followed by bortezomib maintenance for newly diagnosed mantle cell Iymphoma: SWOG S0601. British Journal of Haematology, 172, 208-218.

Wang, M.L., Rule, S., Martin, P., Goy, A., Auer, R., Kahl, B.S., Jurczak, W., Advani, R.H., Romaguera, J.E., Williams, M.E., Barrientos, J.C., Chmielowska, E., Radford, J., Stilgenbauer, S., Dreyling, M., Jedrzejczak, W.W., Johnson, P., Spurgeon, S.E., Li, L., Zhang, L., Newberry, K., Ou, Z.S., Cheng, N., Fang, B.L., McGreivy, J., Clow, F., Buggy, J.J., Chang, B.Y., Beaupre, D.M., Kunkel, L.A. \& Blum, K.A. (2013) Targeting BTK with Ibrutinib in Relapsed or Refractory MantleCell Lymphoma. New England Journal of Medicine, 369, 507-516.

Zinzani, P.L., Vose, J.M., Czuczman, M.S., Reeder, C.B., Haioun, C., Polikoff, J., Tilly, H., Zhang, L., Prandi, K., Li, J. \& Witzig, T.E. (2013) Long-term follow-up of lenalidomide in relapsed/refractory mantle cell lymphoma: subset analysis of the NHL-003 study. Annals of Oncology, 24, 2892-2897.

Table I. Study drugs by dose, days administered, and route.

\begin{tabular}{lccc}
\hline Cycle 1 and 3 & Dose & Days & Route \\
\hline \hline Rituximab & $375 \mathrm{mg} / \mathrm{m}^{2}$ & 1 & IV \\
Cyclophosphamide & $300 \mathrm{mg} / \mathrm{m}^{2}$ & $2-4$ & IV \\
Doxorubicin & $16.6 \mathrm{mg} / \mathrm{m}^{2}$ & $5-7$ & IV \\
Vincristine & $1.4 \mathrm{mg} / \mathrm{m}^{2}(\mathrm{cap} 2)$ & 5 and 12 & IV \\
Dexamethasone & $40 \mathrm{mg}$ & $2-5,12-15$ & IV or PO \\
\hline Cycle 2 and 4 & Dose & Days & Route \\
\hline Rituximab & $375 \mathrm{mg} / \mathrm{m}^{2}$ & 1 & IV \\
Methotrexate & $200 \mathrm{mg} / \mathrm{m}^{2} \mathrm{IV}$ over $2 \mathrm{~h}$ & 2 & IV \\
& $800 \mathrm{mg} / \mathrm{m}^{2} \mathrm{IV}$ over $22 \mathrm{~h}$ & & IV \\
Cytarabine & $3 \mathrm{~g} / \mathrm{m}^{2}$ & $3-4$ & IV \\
Rituximab & $375 \mathrm{mg} / \mathrm{m}^{2}$ & 1 & \\
\hline
\end{tabular}

This article is protected by copyright. All rights reserved 
Table II. Patient characteristics

\begin{tabular}{|c|c|c|c|}
\hline Characteristics & $\mathrm{RH}(\mathrm{N}=17)^{*}$ & $\mathrm{RB}(\mathrm{N}=35)$ & P-value \\
\hline Age, years & $59(44-66)$ & $57(33-64)$ & 0.23 \\
\hline Male/female & $9(53 \%) / 8(47 \%)$ & $32(91 \%) / 3(9 \%)$ & 0.003 \\
\hline \multicolumn{4}{|l|}{ Performance status } \\
\hline 0 & $11(65 \%)$ & $26(74 \%)$ & \multirow[t]{2}{*}{0.52} \\
\hline 1 & $6(35 \%)$ & $9(26 \%)$ & \\
\hline \multicolumn{4}{|l|}{ Disease stage } \\
\hline III & $1(5.9 \%)$ & $3(8.5 \%)$ & \multirow[t]{2}{*}{1.00} \\
\hline IV & $16(94.1 \%)$ & $32(91.4 \%)$ & \\
\hline Bulky disease & $1(6 \%)$ & $3(9 \%)$ & 1.00 \\
\hline B symptoms & $6(35 \%)$ & $10(29 \%)$ & 0.75 \\
\hline Bone marrow involvement & $14(82 \%)$ & $30(86 \%)$ & 1.00 \\
\hline Extranodal involvement & $15(88 \%)$ & $32(91 \%)$ & 1.00 \\
\hline Elevated lactate dehydrogenase & $5(29 \%)$ & $9(26 \%)$ & 1.00 \\
\hline \multicolumn{4}{|l|}{$\operatorname{Ki67}(\mathrm{N}=46)$} \\
\hline$<10 \%$ & $20 \%$ & $10 \%$ & \multirow{3}{*}{0.58} \\
\hline $10-30 \%$ & $60 \%$ & $68 \%$ & \\
\hline$>30 \%$ & $20 \%$ & $22 \%$ & \\
\hline MIPI score & & & 1.00 \\
\hline Intermediate/high & $6(35 \%)$ & $13(37 \%)$ & \\
\hline Low risk & $11(65 \%)$ & 22 (63\%) & \\
\hline
\end{tabular}

MIPI, mantle cell lymphoma International Prognostic Index; RB, rituximab plus bendamustine; $\mathrm{RH}$, R-HyperCVAD (rituximab with hyperfractionated cyclophosphamide, vincristine, doxorubicin and dexamethasone, alternating with high dose cytarabine and methotrexate)

*18 patients randomized to $\mathrm{RH}$. However, 1 patient randomized to $\mathrm{RH}$ on the day $\mathrm{RH}$ arm was closed. That patient did not receive $\mathrm{RH}$ and instead received $\mathrm{RB}$ as per investigator choice. Thus only $17 / 18$ patients were included in analysis.

Table III. Adverse events

\begin{tabular}{lcc}
\hline $\begin{array}{l}\text { Grade 3/4 toxicities (induction } \\
\text { phase) }\end{array}$ & $\mathrm{RH}(\mathrm{N}=17)$ & $\mathrm{RB}(\mathrm{N}=35)$ \\
\hline \hline Anaemia & $59 \%$ & $8.6 \%$ \\
Neutropenia & $65 \%$ & $34 \%$
\end{tabular}

This article is protected by copyright. All rights reserved 


\begin{tabular}{lcc} 
Febrile neutropenia & $29 \%$ & $14 \%$ \\
Thrombocytopenia & $71 \%$ & $17 \%$ \\
Hypokalaemia & $29 \%$ & $5.7 \%$ \\
Hypophosphataemia & $24 \%$ & $2.9 \%$ \\
Hyperglycaemia & $12 \%$ & $0 \%$ \\
ALT increased & $5.9 \%$ & $0 \%$ \\
AST increased & $5.9 \%$ & $0 \%$ \\
Catheter-related infection & $5.9 \%$ & $2.9 \%$ \\
Dehydration & $5.9 \%$ & $0 \%$ \\
Diarrhoea & $5.9 \%$ & $0 \%$ \\
Epistaxis & $5.9 \%$ & $0 \%$ \\
Nausea & $5.9 \%$ & $0 \%$ \\
Rash & $5.9 \%$ & $2.9 \%$ \\
Syncope & $5.9 \%$ & $0 \%$ \\
\hline ALT, alanis
\end{tabular}

$\mathrm{ALT}$, alanine transaminase; AST, aspartate transaminase; $\mathrm{RB}$, rituximab plus bendamustine; $\mathrm{RH}$, R-HyperCVAD (rituximab with hyperfractionated cyclophosphamide, vincristine, doxorubicin and dexamethasone, alternating with high dose cytarabine and methotrexate)Table IV. Reasons for discontinuations

\begin{tabular}{lcc}
\hline $\begin{array}{l}\text { Reasons for discontinuing } \\
\text { treatment/stopping before Auto-HCT }\end{array}$ & $\begin{array}{c}\mathrm{RH}(\mathrm{N}=17) \\
12 / 17\end{array}$ & $\begin{array}{c}\mathrm{RB}(\mathrm{N}=35) \\
14 / 35\end{array}$ \\
\hline \hline Failure to collect stem cells & 5 & 2 \\
Thrombocytopenia & 5 & \\
Patient choice & & 4 \\
Progressive disease & 1 & 2 \\
Pancytopenia & & 1 \\
Neutropenia & & 1 \\
Allergy & & 1 \\
Seizure & & 1 \\
Insurance denial & 1 & 2 \\
Others & & \\
\hline Auto-HCT, autologous haematopoietic stem cell transplantation; RB
\end{tabular}

Auto-HCT, autologous haematopoietic stem cell transplantation; RB, rituximab plus bendamustine; $\mathrm{RH}$, R-HyperCVAD (rituximab with hyperfractionated cyclophosphamide, vincristine, doxorubicin and dexamethasone, alternating with high dose cytarabine and methotrexate)

Figure 1. Study schema.

This article is protected by copyright. All rights reserved 
Ara- $\mathrm{C}=$ cytarabine; BEAM = carmustine $[\mathrm{BCNU}] /$ etoposide/cytarabine/melphalan; $\mathrm{CBV}$ = carmustine $[\mathrm{BCNU}]$, cyclophosphamide, etoposide; Cy, cyclophosphamide; HCVAD = ; HyperCVAD (hyperfractionated cyclophosphamide, vincristine, doxorubicin and dexamethasone, alternating with high dose cytarabine and methotrexate) MTX, = methotrexate; $\mathrm{PR}$ = partial response; $\mathrm{R}$ = rituximab; $\mathrm{TBI}$ = total body irradiation; $\mathrm{VP16}=$ etoposide.

\section{Figure 2. Two-year progression-free survival}

HCVAD/MTX/Ara-C = HyperCVAD (hyperfractionated cyclophosphamide, vincristine, doxorubicin and dexamethasone, alternating with high dose cytarabine and methotrexate); $\mathrm{R}$ = rituximab.

\section{Figure 3. Two-year overall survival}

HCVAD/MTX/Ara-C = HyperCVAD (hyperfractionated cyclophosphamide, vincristine, doxorubicin and dexamethasone, alternating with high dose cytarabine and methotrexate); $\mathrm{R}=$ rituximab.

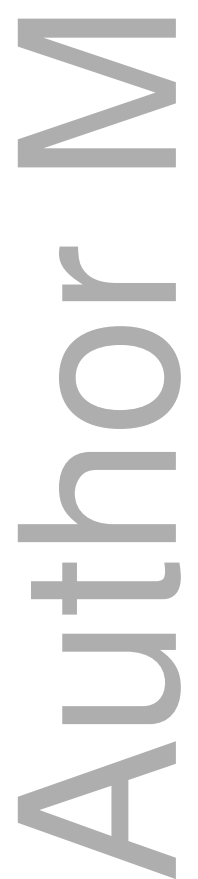




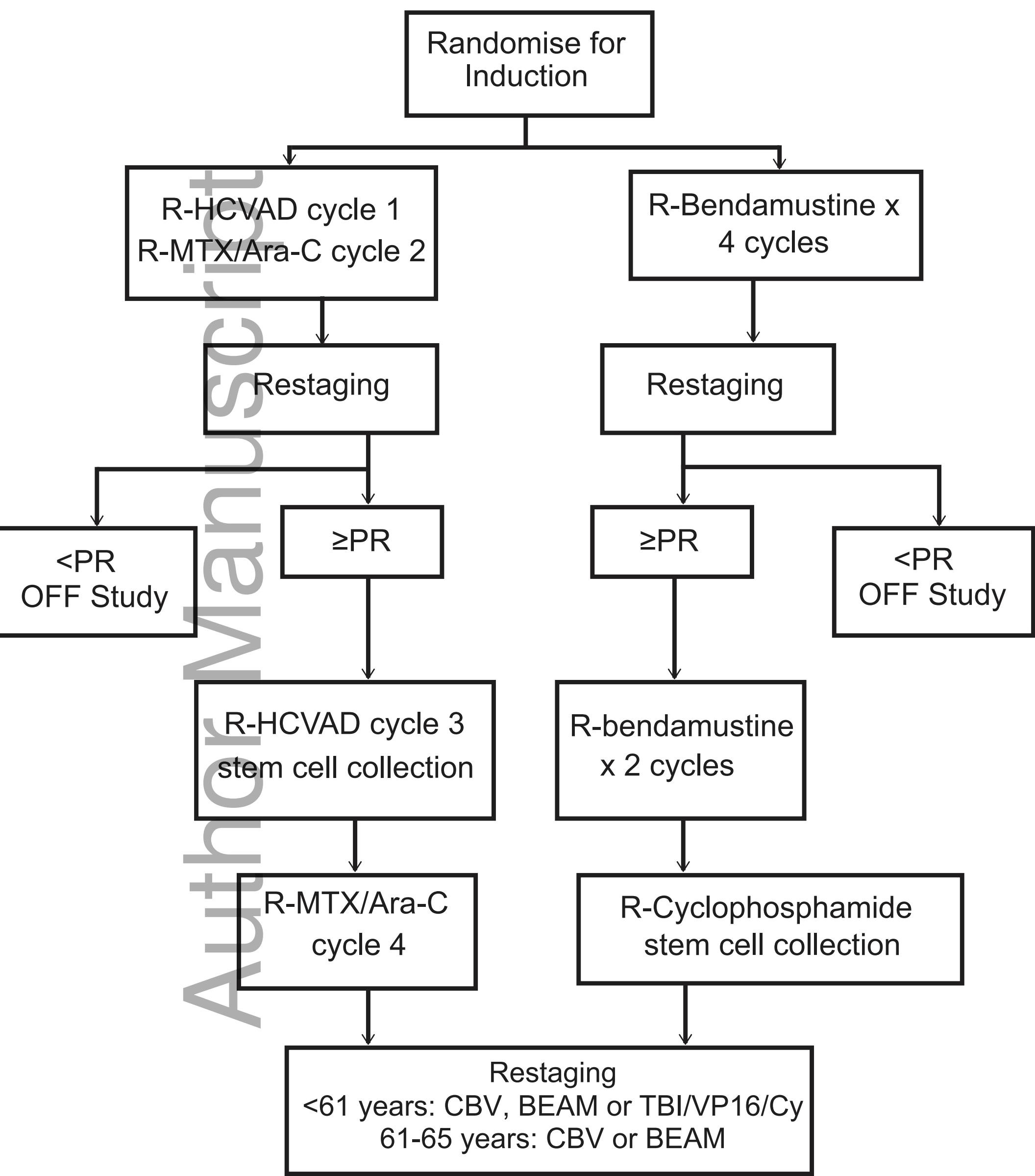

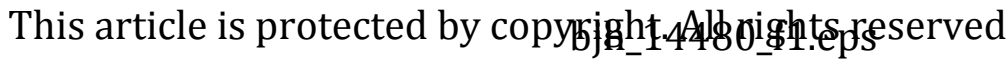




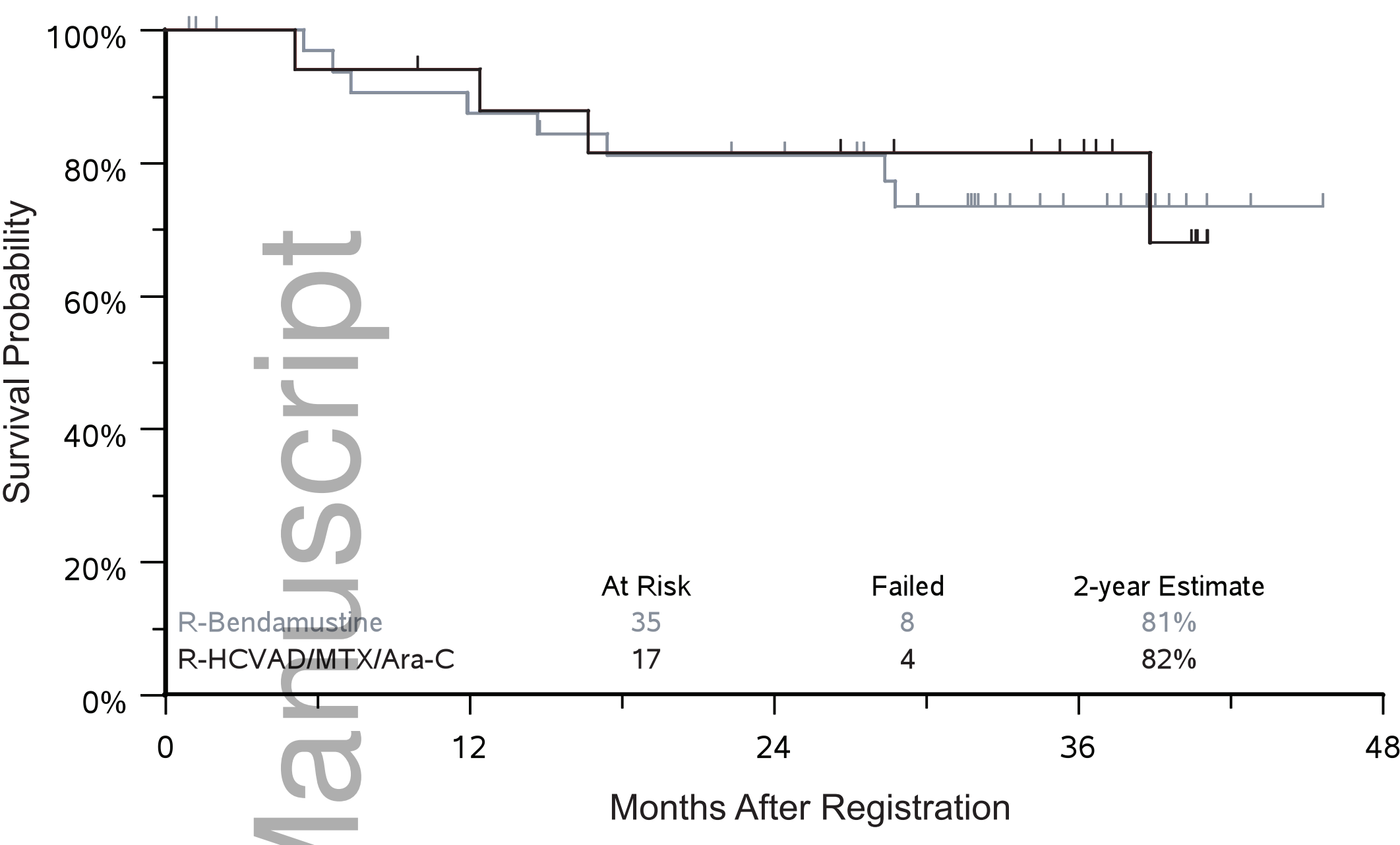

bjh_14480_f2.eps 


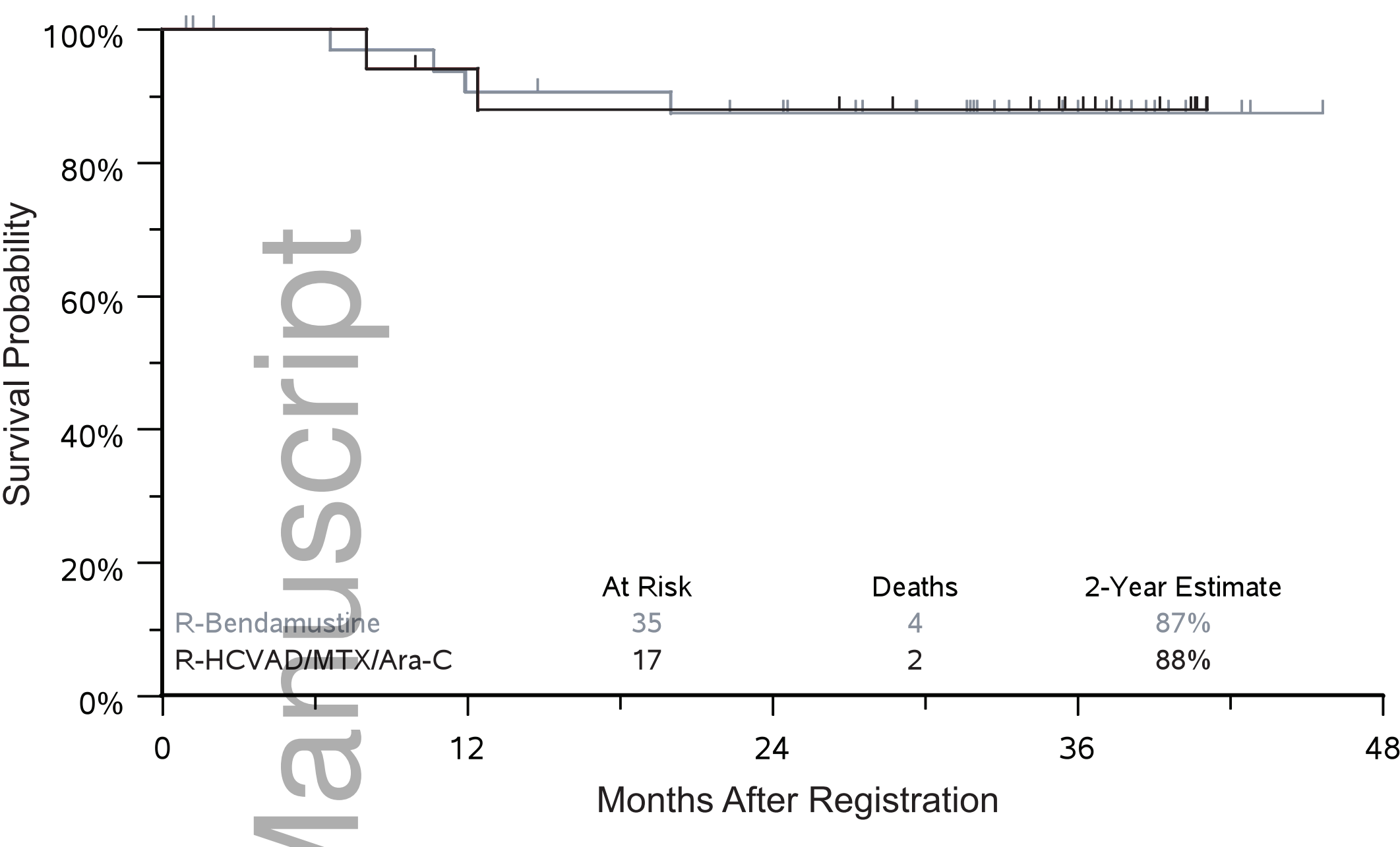

bjh_14480_f3.eps 